Ann. Biol. anim. Bioch. Biophys., I968, 8 (2), 225-233.

\title{
DU RÔLE DE L'HYPOPHYSE ET DES OVAIRES DANS LA BIOSYNTHÈSE DES GSTROGÈNES AU COURS DE LA GESTATION CHEZ LA TRUIE
}

\author{
J. FÈVRE, P.-C. LÉGLISE et P. ROMBAUTS \\ avec la collaboration technique d'Odette REYNAUD \\ Station centrale de Physiologie animale, \\ Station centrale de Recherche de Nutrition, \\ Centre national de Recherches zootechniques, 78 -Jouy-en-Josas \\ Institut national de la Recherche agronomique
}

\section{SOMMAIRE}

Dans l'intention de connaître l'origine de la synthèse des oestrogènes durant la gestation chez la Truie, nous avons suivi l'excrétion urinaire d'œestrone chez deux truies hypophysectomisées le $2^{\mathrm{e}}$ ou le $3^{\mathrm{e}}$ jour de la gestation et chez deux truies ovariectomisées après la saillie. La gestation a été maintenue par l'injection quotidienne de $300 \mathrm{mg}$ de progestérone. Malgré l'absence d'ovaires ou d'hypophyse, l'excrétion urinaire d'œstrone présente une cinétique identique à celle que l'on peut trouver chez des animaux gestants intacts, avec un premier maximum précoce à la fin du premier mois de gestation, puis, à partir du $80^{\mathrm{e}}$ jour, les valeurs maximales de la gestation.

Des résultats, il ressort que :

- dès la fin du premier mois de gestation la synthèse des œestrogènes n'est pas ovarienne ;

- l'élimination d'œstrone semble proportionnelle au nombre de foetus dans des conditions physiologiques identiques ; maternelle.

- la synthèse des œestrogènes pendant la gravidité n'est pas sous la dépendance de l'hypophyse

On peut conclure que, au cours de la gestation chez la Truie, l'ensemble fœto-placentaire possède les activités enzymatiques nécessaires à la synthèse des œstrogènes.

\section{INTRODUCTION}

L'excrétion urinaire d'œstrogènes chez la Truie gestante a été bien étudiée dans les conditions normales de la gestation, notamment par RoMBAUTS (I962), LUNAAs (I962) et RAESIDE (I 963 ). Ces auteurs ont mis en évidence l'importance de l'excrétion d'œstrone et la présence d'un premier maximum d'élimination à la fin du premier mois de gestation. ROMBAUTS, puis RAESIDE ont montré que l'excrétion n'aug- 
mente ensuite de nouveau qu'à partir du $80^{\mathrm{e}}$ jour de gestation pour se maintenir à de fortes valeurs jusqu'à la parturition. En nous reportant à nos résultats antérieurs sur truies intactes (RombauTs, I962), nous trouvons pour les truies 2245 et I25I gestantes de II et I2 fotus, une excrétion journalière moyenne de $0,54 \mathrm{mg}$ d'œstrone par porcelet et pour une autre gestation de la Truie I25I avec rg fœtus, $0,58 \mathrm{mg}$. Les valeurs de RAESIDE (I963) pour une truie portant I4 fœtus donnent une moyenne de 0,7 I $\mathrm{mg}$ par porcelet, valeur comparable aux nôtres si l'on effectue la correction pour les pertes au cours de l'analyse. Chez l'animal intact, compte tenu de l'élimination par la voie fécale et des métabolites autres que l'œstrone, on peut donc estimer l'excrétion journalière d'cestrogènes en fin de gestation chez la Truie à environ I mg par fœtus.

L'origine des fortes quantités d'œstrogènes est encore mal connue, particulièrement en début de gestation, car 1'on n'avait encore jamais observé d'augmentation de la sécrétion à cette époque chez les autres Mammifères.

Par différents critères, notamment des réductions utérines en début de gestation et des ovariectomies en fin de gestation, l'un de nous a montré que la synthèse des œstrogènes, était vraisemblablement d'origine fœto-placentaire et non ovarienne, chez la Truie (Rombauts, I964). Nous avons cherché à confirmer ces résultats en évaluant la sécrétion d'œstrogènes chez des truies privées d'hypophyse ou d'ovaires dès les premiers jours de la gestation. Nous avons adopté comme critère de la sécrétion, la mesure de l'excrétion urinaire d'œstrone ; cette hormone représente en effet, 67 p. Ioo des métabolites des œstrogènes de 1'urine et son excrétion se fait à 95 p. I00 par la voie urinaire chez la Truie (ROMBaU'Ts, TERQUr et FÈvRE, I966).

\section{MATÉRIEL, ET MÉTHODES}

\section{Matériel}

Deux truies de race Large-White furent hypophysectomisées, l'une le $\mathbf{2}^{\mathbf{e}}$ jour, l'autre le $3^{\mathbf{e}}$ jour après la saillie. L'intervention chirurgicale fut réalisée par voie transfrontale sus-orbitaire selon la technique décrite par du Mesnil du BuIsson, LÉGlise et ChoDkiEwiCz (1964). Deux truies de race Large-White furent ovariectomisées, l'une 5 jours après la saillie, l'autre 8 jours après la saillie. Les animaux opérés furent maintenus en cage à métabolisme dans une salle climatisée $\left(25^{\circ} \mathrm{C} \pm \mathrm{I}^{\circ} \mathrm{C}\right)$. Le placenta ne sécrétant pas de progestérone chez la Truie, chaque animal reçut quotidiennement, pendant toute la gestation, une injection intramusculaire de $300 \mathrm{mg}$ de progestérone.

Les truies hypophysectomisées subirent une césarienne, la parturition ne s'étant pas produite spontanément le II $4^{\mathrm{e}}$ jour de gestation. L'autopsie de ces animaux a permis de vérifier que l'hypophysectomie avait été totale. Chez la Truie, l'hypophyse peut être enlevée facilement in toto. Elle ne présente en effet aucune adhérence.

Les urines, séparées des fèces, furent recueillies quantitativement dans des bacs en polyéthylène avec une quantité d'acide chlorhydrique suffisante pour obtenir un $\mathrm{pH}$ final de 4 à 5 , soit quotidiennement, soit en réalisant des échantillons moyens de 2 ou 3 jours en prélevant des parties aliquotes. Les échantillons ainsi récoltés furent conservés à $-15^{\circ} \mathrm{C}$ dans les cas où les dosages ne purent être effectués immédiatement. Cette conservation au froid et en milieu acide n'entraîne pas de pertes d'œstrogènes.

\section{Méthodes de dosage}

Les œstrogènes ont été dosés selon une méthode chromatographique dérivée de celle de BrowN (1955). Nous avons apporté à la méthode originale les modifications suivantes :

- I'hydrolyse acide des sulfo- et glucuroconjugués fựt remplacée par l'hydrolyse enzymatique 
(JAYLE et al., I959) : l'échantillon d'urine est amené à $\mathrm{pH} 4,8$; on ajoute ensuite un tampon acétate $2 \mathrm{M}$ (I/IO du volume de l'échantillon). L'échantillon est mis alors à incuber $22 \mathrm{~h}$ environ à $37^{\circ} \mathrm{C}$ avec du suc digestif d'Helix pomatia (I ooo unités Fishman de $\beta$-glucuronidase et 8000 unités Roy de sulfatase par $\mathrm{ml}$ d'urine). A l'aide de stéroïdes conjugués radioactifs, nous avons vérifié que l'hydrolyse était totale.

- Pour la purification des extraits urinaires, la saponification préconisée par BAULD (I956) puis par BROWN, BulbROOK et GREeNWOOD (1957) a été adoptée.

Les déterminations colorimétriques furent réalisées, avec extraction de la coloration rose de Kober par un solvant organique, en l'occurence une solution à 2 p. 10o de para-nitrophénol dans le tétrachlorure d'acétylène suivant la technique d'ITTRICH (I958 et r960). Les lectures ont été effectuées à 3 longueurs d'onde afin d'appliquer la correction d'Allen : 510-540 et $570 \mathrm{~m} \mu$.

Ces modifications ont amélioré la sensibilité de la méthode, ainsi abaissée à $5 \mu \mathrm{g}$ d'œstrogènes excrétés en $24 \mathrm{~h}$. Les valeurs sont données sans correction pour les pertes au cours du dosage et peuvent être ainsi comparées directement à nos résultats antérieurs (ROMBAUTS, I962). Le pourcentage de récupération de l'œstrone par cette méthode est en moyenne de $85 \mathrm{p}$. Ioo.

\section{RÉSULTATS}

L'injection journalière de $300 \mathrm{mg}$ de progestérone a permis le maintien de l'état gravidique. Les truies hypophysectomisées ont eu, l'une $\left(n^{0} 2403\right) 8$ porcelets vivants, 1'autre $\left(n^{\circ} 2532\right)$ I porcelet vivant. Une truie ovariectomisée ( $\left(n^{\circ} 2215\right)$ a donné naissance à 6 porcelets vivants, l'autre $\left(n^{0} 2677\right)$ a eu 4 porcelets morts quelques jours avant la mise bas. La natalité est cependant faible, en particulier pour la truie 2215, qui, au cours de trois gestations précédentes, n'avait jamais eu moins de I5 porcelets par portée.

Comme chez l'animal intact, le principal astrogène présent dans l'urine des truies en expérience est l'œestrone (estra I-3-5 (I0) triène-3 ol-I7 one). La sensibilité de la méthode utilisée est insuffisante pour que les mesures des faibles quantités d'œstradiol I7- $\beta$ (estra I-3-5 (I0) triène 3,I7- $\beta$ diol) soient significatives. Aussi nous sommes-nous limités à l'analyse de la fraction " cestrone ".

L'excrétion urinaire d'œstrone chez trois des quatre truies présente la même cinétique que celle des animaux gestants intacts (fig. $x, 2,3$ ). Nous avons trouvé en effet, à la fin du premier mois de gestation, un premier maximum d'excrétion, avec des valeurs supérieures à $2 \mathrm{mg}$ d'oestrone par $24 \mathrm{~h}$.

La dernière truie, hypophysectomisée, n'a pu être mise en expérience qu'à partir du $3^{\mathrm{e}}$ jour de gestation en raison de son état de santé à la suite de l'opération. Les valeurs d'œstrone urinaire pour cette truie diminuent du $30^{\mathrm{e}}$ au $35^{\mathrm{a}}$ jour de gestation, ce qui semble bien montrer que l'on se trouve dans la phase décroissante après le premier maximum d'excretion (fig. 4).

Du $35^{\mathrm{e}}$ au $75^{\mathrm{e}}$ jour de gestation, les valeurs sont faibles, en général inférieures à $50 \mu \mathrm{g}$ par jour. A partir du $75^{\mathrm{e}}$ jour l'excrétion urinaire d'œstrone recommence à. augmenter avec des valeurs maximales au cours des derniers jours de gestation. Cependant, chez la truie 2677 , ovariectomisée (fig. 4) les valeurs ont baissé régulièrement à partir du ro8 jour de gestation jusqu'à la mise bas. Cette baisse peut s'expliquer par la mort des foetus in utero. Ira parturition de la truie 2215 a été prématurée par suite de l'arrêt trop précoce des injections de progestérone (au Io8e jour). Il nous manque donc les valeurs finales en général élevées. 


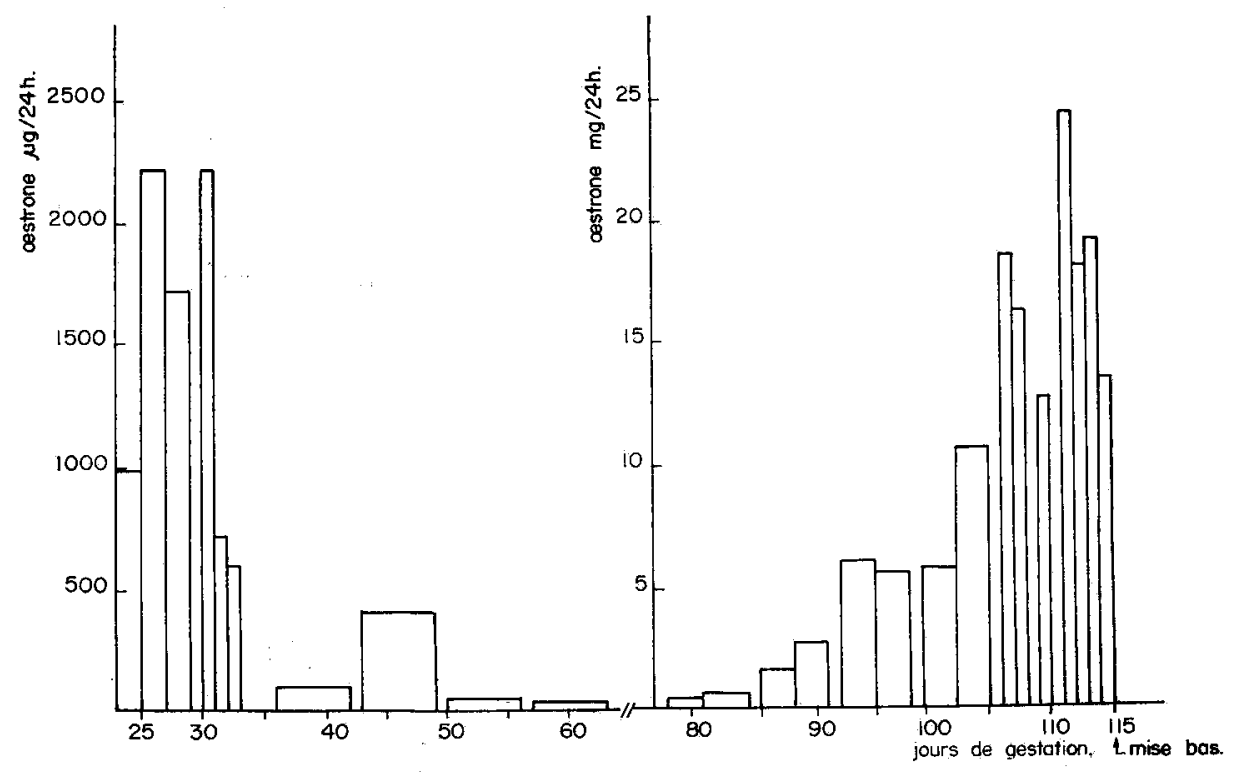

FIG. I. - Excrétion urinaire d'cestrone

Truie $2403-$ Hypophysectomisée

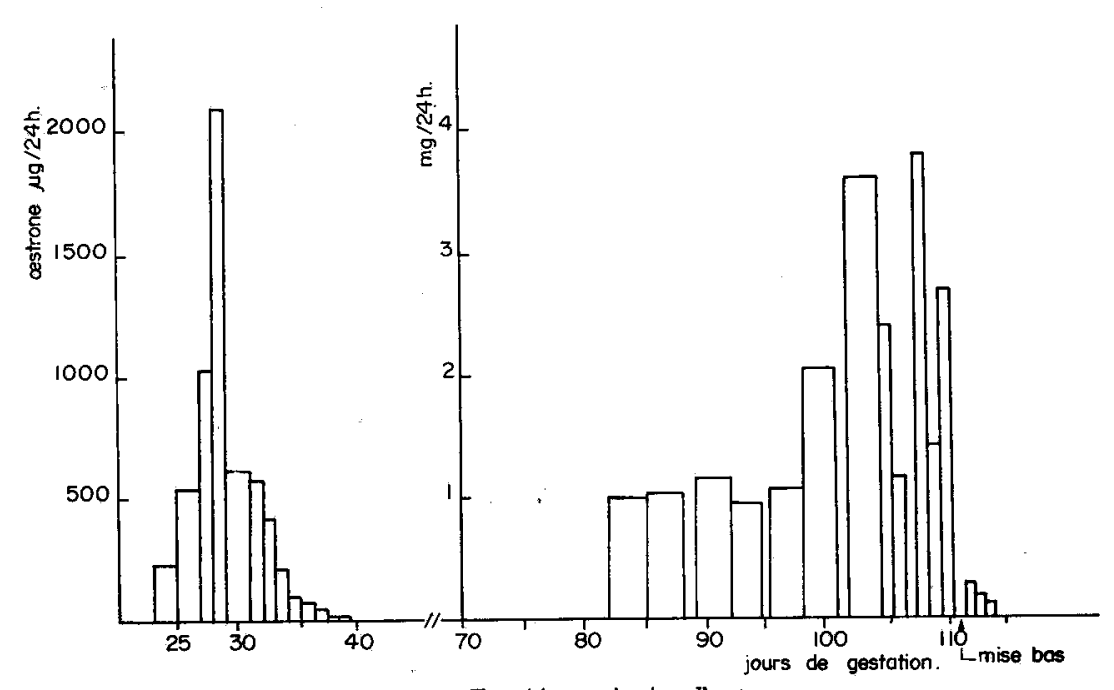

FIG. 2. - Excrétion urinaire d'estrone

Truie 2215 - Ovariectomisée 

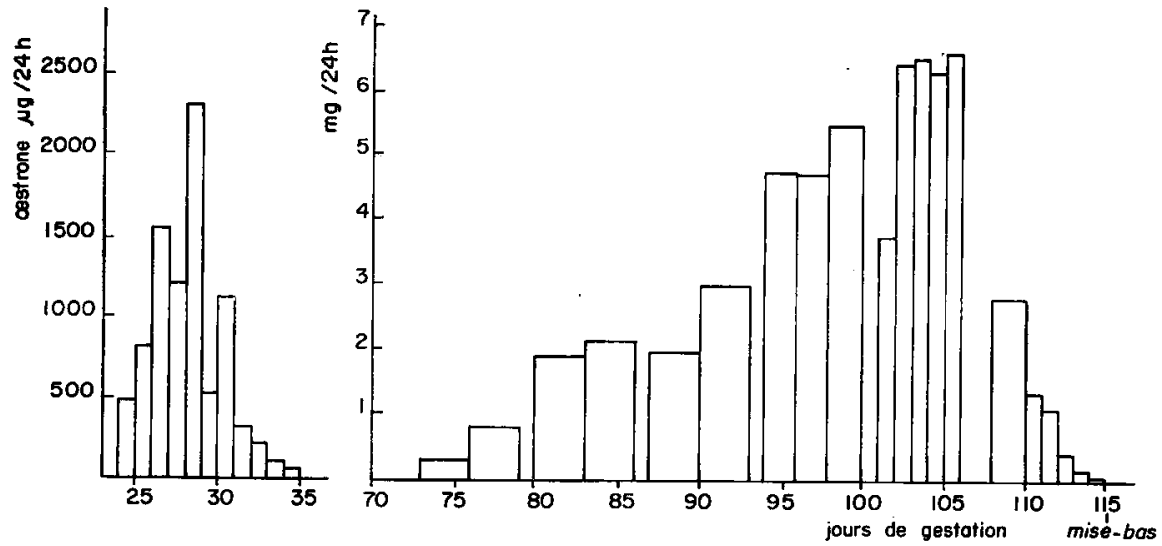

FIG. 3. - Excrétion urinaire d'cestrone

Truie 2677 - Ovariectomisée

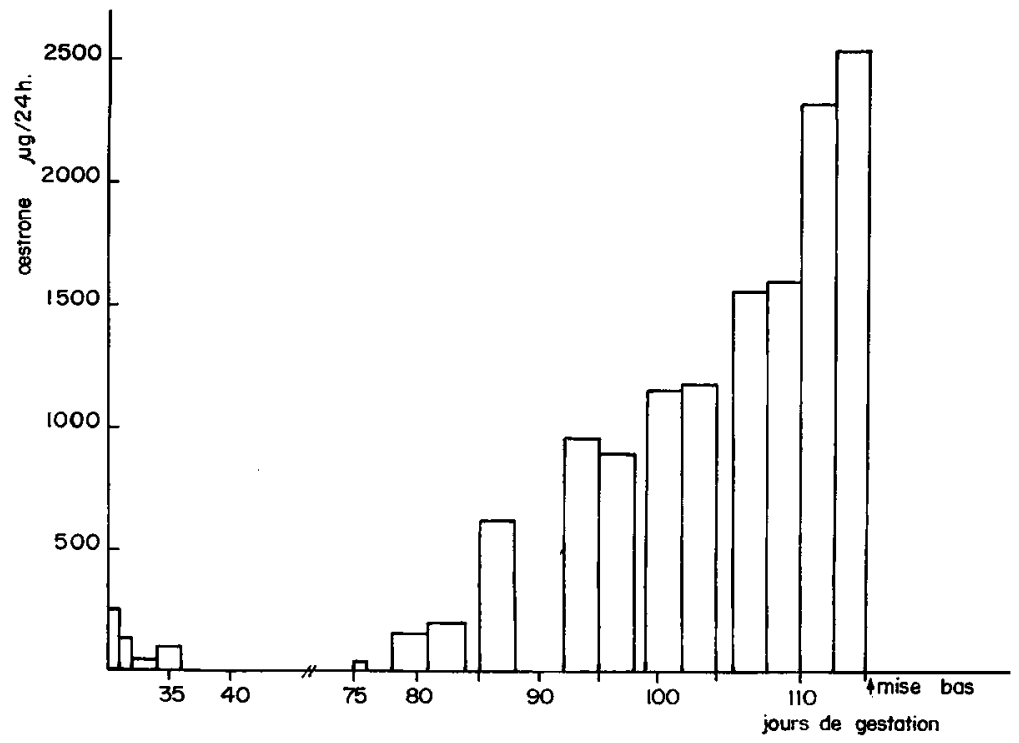

FIG. 4. - Excrétion urinaire d'ostrone

Truie $253^{2}-$ Hypophysectomisée 
L'excrétion urinaire d'œstrone a été suivie après la mise bas chez la truie 2215 , ovariectomisée (tab1. I et fig. 2). Le jour suivant la parturition, 1'excrétion urinaire d'œestrone n'est plus que le r/Io de ce qu'elle était avant et elle chute rapidement les jours suivants pour arriver à $0,02 \mathrm{mg}$ le $4^{\mathrm{e}}$ jour, comme chez l'animal intact (Rombauts, I962).

\section{TABLEAU I}

Excrétion urinaire d'cestrone

(Truie 2215 ovariectomisée)

\begin{tabular}{c|c|c|c}
\hline \hline \multicolumn{2}{c|}{ Avant parturition } & \multicolumn{2}{|c}{ Après parturition } \\
\hline Jours & Estrone (mg/24 h) & Jours & Estrone (mg/24 h) \\
& & & \\
\cline { 3 - 4 }-5 et -6 & 1,77 & +1 & 0,26 \\
-3 et -4 & 3,04 & +2 & 0,18 \\
-1 et -2 & 1,98 & +3 & 0,11 \\
0 & parturition & +4 & 0,02 \\
\hline
\end{tabular}

Si la cinétique de l'excrétion est la même pour les quatre animaux, il en va tout autrement de son amplitude, particulièrement en fin de gestation. Les deux animaux hypophysectomisés ont un maximum d'élimination journalière d'œstrone très différent : 22,7 mg pour la truie 2403 et 2,9 mg pour la Truie 2532 , mais, ramenée à un porcelet, l'excrétion est identique : 2,84 et $2,9 \mathrm{mg}$ respectivement (tabl. 2). Ce fait pourrait permettre de penser qu'il y aurait une proportionnalité entre la sécrétion d'œstrogènes et le nombre de fœtus chez la Truie. Il faudrait évidemment répéter cette expérience sur un nombre plus important d'animaux pour vérifier cette relation.

\section{TABLEAU 2}

Excrétion urinaire d'cstrone en fonction du nombre de foetus (Résultats correspondant au maximum d'excrétion)

\begin{tabular}{|c|c|c|c|c|}
\hline \multirow[b]{2}{*}{ Numéro de l'Animal } & \multicolumn{2}{|c|}{ Hypophysectomisées } & \multicolumn{2}{|c|}{ Ovariectomisées } \\
\hline & 2403 & 2532 & 2215 & 2677 \\
\hline 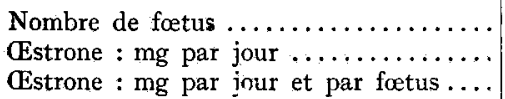 & $\begin{array}{c}8 \\
22,7 \\
2,84\end{array}$ & $\begin{array}{l}1 \\
2,9 \\
2,9\end{array}$ & $\begin{array}{l}6 \\
3,8 \\
0,64\end{array}$ & $\begin{array}{l}4 \\
6,64 \\
1,65\end{array}$ \\
\hline
\end{tabular}

Bien qu'elles n'aient plus d'ovaires, les truies ovariectomisées présentent un premier maximum d'excrétion au $28 \mathrm{e}$ jour de gestation, d'ampleur comparable à celle d'animaux intacts et à celle de l'hypophysectomisée 2403 (2,I et 2,3 mg/24 h). En revanche, en fin de gestation, 1'excrétion urinaire est relativement faible. Le maximum trouvé le Ioge jour de gestation chez l'animal 2215 est de $3,8 \mathrm{mg}$ par $24 \mathrm{~h}$. 
La même-truie au cours d'une gestation antérieure a présenté une excrétion urinaire d'œstrone de $7,96 \mathrm{mg}$ par $24 \mathrm{~h}$ à J. ro8 et de 9,2I mg par $24 \mathrm{~h}$ à J. Iog. L'importance des portées n'était pas la même dans les deux cas : 6 porcelets lors de la gestation après ovariectomie et $\mathrm{I} 7$ porcelets pour 1'autre. Si l'on considère l'excrétion urinaire d'œstrone par $2.4 \mathrm{~h}$ et par porcelet (tabl. 2), aux Io8 et Ioge jours de gestation chez cette truie, on a pour la gestation après ovariectomie, $0,64 \mathrm{mg}$ et pour la gestation normale $0,54 \mathrm{mg}$. Ainsi, au même stade de gestation et à portée égale, nous trouvons des valeurs très voisines chez le même animal, avant et après ovariectomie. Pour la truie 2677 ovariectomisée, nous avons une excrétion maximale de $1,65 \mathrm{mg}$ par jour et par fotus. Cet animal maintenu dans une pièce chaude a reçu de l'eau de boisson à volonté et sa diurèse fut plus abondante que celle des animaux limités en eau.

\section{DISCUSSION}

A la fin du premier mois de gestation, la truie 22 55 présente une excrétion urinaire d'œstrone de 2 , Io $\mathrm{mg}$ par $24 \mathrm{~h}$, valeur peu différente de celle de fin de gestation $(3,04 \mathrm{mg}$ par $24 \mathrm{~h})$. Chez des animaux intacts ou même hypophysectomisés, les valeurs à ces deux périodes sont nettement différentes ( 5 à ro fois plus fortes en fin de gestation). On pourrait penser que l'importance du premier maximum d'excrétion est indépendante de la taille de la portée. Cette hypothèse semble devoir être abandonnée. En effet, Rombauts (I964), mesurant l'excrétion urinaire d'œstrone chez des truies qui avaient subi des réductions utérines au cours de la gestation, a trouvé des valeurs supérieures à celles du cycle oestral (ce qui confirmait 1'état gestatif) mais nettement inférieures à celles que 1'on rencontre dans les gestations des animaux intacts. Il faut plutôt penser qu'il y a eu entre la fin du premier mois de gestation et la parturition, des résorptions de fœtus, hypothèse confirmée par le nombre de corps jaunes présents au moment de l'ovariectomie : les ovaires de la truie 2677 présentaient I3 corps jaunes à la castration alors qu'il n'y a eu que quatre porcelets à la parturition.

Du Mesnil, du Buisson (Ig66) a constaté sur de nombreuses truies que, 8 jours après hypophysectomie, les ovaires n'ont plus de follicules en développement et qu'ils régressent très vite. On peut donc dire qu'au cours de notre expérience, aussi bien chez les animaux hypophysectomisés que chez les animaux ovariectomisés, les œestrogènes présents dans l'urine ont une origine extra-ovarienne. I,a progestérone exogène administrée aux truies ne modifie pas la production d'œstrogènes. En dehors des deux maximums d'excrétion, en effet, nous ne trouvons que très peu d'œstrone alors que les injections de progestérone étaient maintenues. RombaUTs (I964), après avoir ovariectomisé des truies vers le Ioo $^{\mathrm{e}}$ jour de gestation maintenait la gestation par l'injection de $200 \mathrm{mg}$ de progestérone. Deux animaux ont avorté. Chez ces truies, bien que ce fût en fin de gestation, l'excrétion urinaire d'œstrone était très faible. Enfin, Arnsworth et RYAN (I966) ont montré que in vitro des préparations de divers placentas de mammifères, en particulier de truies, n'étaient pas capables de synthétiser des oestrogènes en quantités détectables après incubation avec des stéroïdes 
en $\mathrm{C}_{21}$ et principalement avec la progestérone, ce qui laisse supposer une activité r7-20 desmolase placentaire très faible ou même nulle.

La biosynthèse des oestrogènes au cours de la gestation chez la Truie a donc bien lieu dans l'ensemble fœeto-placentaire, même à la période précoce du premier mois. Bien que le placenta de Truie soit de type épithéliochorial et qu'il soit incapable de synthétiser la progestérone nécessaire au maintien de la gestation, il possède des activités enzymatiques permettant la synthèse des œstrogènes. L'activité enzymatique du placenta de Truie a d'ailleurs été mise directement en évidence in vitro par AINSWORTH et RYAN (I966).

Nous avons vu que la quantité d'œestrogènes excrétés semblait proportionnelle au 'nombre de jeunes par portée et qu'en cas de gestation normale les valeurs de l'excrétion urinaire d'oestrone se situaient entre 0,5 et $0,7 \mathrm{mg}$ par jour et par fœetus. Cependant une augmentation de la diurèse semble augmenter l'élimination des œstrogènes, ainsi que nous l'avons trouvé chez les animaux hypophysectomisés et chez un animal ovariectomisé.

Chez les animaux hypophysectomisés, nous avons trouvé 2,9 mg par porcelet, soit trois fois plus que chez les animaux intacts. Les truies hypophysectomisées ont un diabète insipide donc une diurèse élevée (ro à 15 litres d'urines par jour, soit deux à trois fois plus que la diurèse normale). I a modification du fonctionnement rénal entraîne peut-être une accélération de l'élimination des stéroïdes œestrogènes, ce qui pourrait expliquer que la synthèse d'hormone s'accroisse. Cependant, il semble que ce ne soit pas le seul facteur qui intervienne dans l'excrétion accrue d'œstrone par les animaux hypophysectomisés. D'autres hypothèses peuvent aussi expliquer ce phénomène. Quel qu'en soit le mécanisme, cette augmentation de l'excrétion d'œstrogènes chez l'animal hypophysectomisé, si elle se confirme sur d'autres animaux, indique soit une synthèse accrue, soit que l'excrétion chez les animaux intacts ne correspond qu'à une partie des hormones sécrétées, part qui pourrait être modifiée par le fonctionnement hypophysaire et rénal.

Les hormones hypophysaires sont indispensables chez la Truie pour induire la sécrétion de progestérone des corps jaunes nécessaires au maintien de la gestation (ANDERSON et al., I965). Les résultats obtenus montrent que, si l'intégrité du fœetus et du placenta est respectée, la biosynthèse des œstrogènes s'effectue normalement, indépendamment de toute stimulation hypophysaire maternelle.

Cette expérience ne permet pas de connaître les rôles respectifs du foetus et du placenta dans la biosynthèse des oestrogènes, ni de savoir si cette synthèse peut s'effectuer à partir d'acétate ou de cholestérol, ou si elle nécessite des précurseurs stéroïdes plus proches dans la voie de la biosynthèse. On peut penser que ces précurseurs sont, comme pour l'espèce humaine, des stérö̈des surrénaliens d'origine maternelle ou fœtale.

Nous poursuivrons donc cette étude par des essais de dissociations foeto-placentaires et des injections de précurseurs radioactifs, notamment la déhydroépiandrostérone et son conjugué sulfate, la $\Delta_{4}$-androstènedione, la testostérone, administrés soit dans le compartiment maternel, soit dans le compartiment foetal. 


\section{SUMMARY}

\section{ROLE OF THE PITUITARY AND OVARIES IN CESTROGEN BIOSYNTHESIS IN THE PREGNANT SOW}

The origin of œstrogen synthesis during the pregnancy of the sow was studied. The urinary excretion of cestrone was estimated on four sows : two were hypophysectomized and two were ovariectomized at the beginning of their pregnancies. The four sows were intramuscularly injected with $300 \mathrm{mg}$ of progesterone per day to prevent abortion.

In spite of the absence of ovaries and pituitary, the urinary excretion of cstrone followed a pattern similar to that of intact animals (fig. I, 2, 3, 4). An excretory peak (2.10 $\mathrm{mg}$ per day) was recorded at the end of the first month. The highest values were reached towards the end of gestation after day 80 . In both hypophysectomized sows, according to the number of fotuses borne, the excretion was remarkably high (2.9 $\mathrm{mg}$ per day and per fœetus).

The urinary excretion of œstrone decreased sharply just after farrowing (table I and fig. 3).

The results of this experiment led us to the following conclusions :

- The urinary oestrogens present, from the end of the first month of pregnancy on, are not of ovarian origin. conditions.

- Estrone excretion seems related to the number of fœtuses under identical physiological

- The synthesis of œestrogens during pregnancy is not under control of the maternal pituitary.

It can be concluded that, in the pregnant sow, the placenta and the fœetus are in possession of all the necessary enzymes and partake in the œstrogen synthesis. They may start from the initiating metabolite steroids of maternal or foetal origin.

\section{RÉFÉRENCES BIBLIOGRAPHIQUES}

AINSWORTH L., RYAN K. J., I 966. Steroid hormone transformations by endocrine organs from pregnant mammals. I. Estrogens biosynthesis by mammalian placental preparation in vitro. Endocrinology, 79, $875-883$.

Anderson L. L., Léglise P. C., du Mesnil du Buisson F., Rombauts P., I965. Interaction des hormones gonadotropes et de l'utérus dans le maintien du tissu lutéal ovarien chez la 'Truie. $C . R$. Acad.Sci., Paris, 261, 3675-3678.

BAuld W. S., 1956. A method for the determination of ostriol, ostrone and cestradiol I7- $\beta$ in human urine by partition chromatography and colorimetric estimation. Biochem. J., 63, 488-495.

Brown J. B., I955. A chemical method for the determination of cestriol, cestrone and cestradiol in human urine. Biochem. J., 60, 185-193.

Brown J. B., Bulbrook R. D., Greenwood F. C., I957. An evaluation of a chemical method for the estimation of œstriol, œstrone and œestradiol $\mathrm{r} 7-\beta$ in human urine. J.endocrinol., 16, 41-48.

ITTRICH G., 1958. Eine neue Methode zur chemischen Bestimmung der östrogen Hormone im Harn. HoppeSeylers Z. Physiol. Chemie., 312, I-14.

ITTRICH G., Ig6o. Untersuchungen über die Extraktion des roten Kober-Farbstoffe durch organische Lösungsmittel zur Östrogensbestimmung im Harn. Acta Endocr., 35, 34-48.

Jayle M. F., Scholler R., Jarrige P., Metay S., 1959. Hydrolyse des phénolstéroïdes conjugués urinaires. Bull. Soc. Chim. Biol., 41, $1593-1603$.

LunaAs T., 1962. Urinary cestrogen levels in the Sow during œestrous cycle and early pregnancy. J. Reprod. Fertil., 4, 13-20.

Du Mesnil du Buisson F., Ltelise P. C., Chodkiewicz J. P., I964. Technique de l'hypophysectomie par voie transfrontale sus-orbitaire chez le Porc. Ann. Biol. anim. Biochim. Biophys., 4, 229-237.

Du Mesnil du Buisson F., ig66. Communication personnelle.

RAEside J. I., 1963. Urinary œstrogen excretion in the Pig during pregnancy and parturition. J. Reprod. Fertil., 6, 427-431.

Rombauts P., 1962. Excrétion urinaire d'œstrogènes chez la Truie pendant la gestation. Ann. Biol. anim. Biochim. Biophys., 2, 151-1 56.

Rombauts P., I 964 . Lieu de synthèse des hormones stéroïdes ostrogènes pendant la gestation de la Truie. C. R. Acad. Sci. Paris, 258, 5257-5259.

Rombauts P., Terqui M., Fivre J., 1966. Site of estrogens production and route of excretion in domestic animals. In Second International Congress on hormonal sieroids, Milan, I966, 283, Excerpta medica Foundation, Amsterdam. 